\title{
Single Phase Voltage Source Inverter Photovoltaic Application
}

\author{
J. Bauer
}

\begin{abstract}
Photovoltaic applications have been developing and spreading rapidly in recent times. This paper describes the control strategy of the Voltage Source Inverter that is the important tail end of many photovoltaic applications.

In order to supply the grid with a sinusoidal line current without harmonic distortion, the inverter is connected to the supply network via a L-C-L filter. The output current is controlled by the hysteresis controller. To improve the behaviors of the L-C-L filter, active damping of the filter is being used. This paper discusses controller design and simulation results.
\end{abstract}

Keywords: voltage source inverter, L-C-L filter, hysteresis controller, pulse width modulation.

\section{Introduction}

Increasing efforts are being made nowadays to use renewable energy sources. Processing the energy obtained from sun, wind or water is coming to the fore. The energy supplied by these sources does not have constant values, but fluctuates according to the surrounding conditions (intensity of sun rays, water flow, etc.). These supplies are therefore supplemented by additional converters. The most used types are inverters or DC/DC converters. The area of high power converters for solar application is already covered by industrial manufacturers. However, the area of low power devices is not fully covered. These converters are mostly built from commercially produced parts that can perform demanded functions, but they are not developed for this type of application and therefore the efficiency of the whole system is low. Low power devices are important in applications where there is no voltage grid present and electric power is required (mountains, desert expeditions, etc.)

The simplified block structure of the investigated system is shown in Fig. 1. A DC/DC converter with an MPPT (Maximum Power Point Tracker) is connected to the solar array. A second DC/DC converter is connected to the output of this converter. The second converter raises the voltage acquired from the solar system to the voltage level demanded by the VSI.

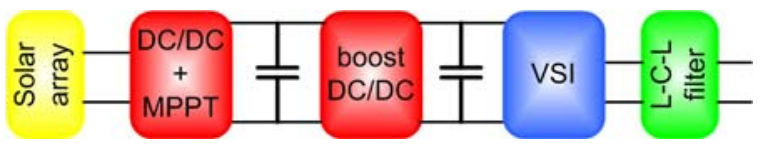

Fig. 1: Block diagram of the system

\section{DC voltage control}

The power produced by solar arrays depends on surrounding conditions, e.g. temperature $\vartheta$ and intensity of sun exposure $E$. The solar array output power is not therefore constant. The output power also depends on the withdrawn current and voltage. (Fig. 2)

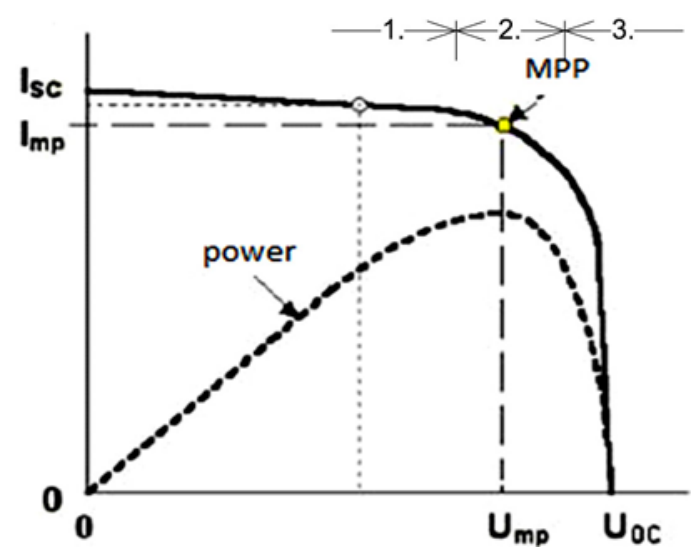

Fig. 2: Output characteristic of the solar cell and the maximum power point tracking principle

The MPPT control algorithm ensures that the solar array operates at its optimal point and that it delivers maximal power. The algorithm periodically changes the duty cycle of the converter in the defined hysteresis range, it increases the duty cycle, it decreases the duty cycle, and it compares the powers delivered by the solar cell. Then it chooses the duty cycle that corresponds with the maximal delivered power. If the converter operates on the left side of the MPP (area 1.), the algorithm will decrease the duty cycle. When it is operating on the right (area 3.) of the MPP, the controller will increase the duty cycle. If the converter is operating at MPP (area 2.), the power obtained on both sides will be lower and the duty cycle remains the same.

The second DC/DC converter is a boost type full bridge with galvanic insulation. The converter works with a fixed duty cycle and boosts the input voltage to the voltage level required by the inverter. For a single phase VSI, $400 \mathrm{~V}$ should be enough. 


\section{Voltage Source Inverter}

The VSI is used for converting energy from DC to AC voltage. The detailed scheme of the inverter is shown in the Fig. 3. The power part of the inverter is made of four MOSFETs and the L-C-L filter is connected to the output of the inverter. This filter ensures the sinusoidal shape of the output current.

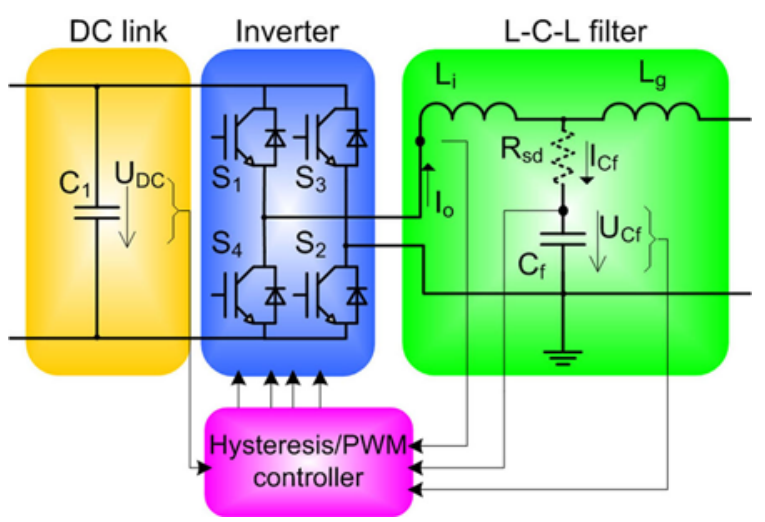

Fig. 3: Inverter diagram

The inverter in this application can operate in two different modes. Firstly, it can operate in the so-called "island mode", which means that the converter acts as a voltage source and supplies some devices with sinusoidal voltage of the common network parameters. PWM control is used for this purpose. The second possible operation mode delivers current to the supply network. PWM control is not suitable for this operation mode and the current hysteresis control is therefore used.

\subsection{Current control}

The simplest hysteresis controller can be realized as a simple bang-bang regulator. The actual value of the output current is controlled in order to remain in a defined area. This method is fast and simple, and provides good results with an L-C-L filter. The only problem is the variable switching frequency of the semiconductor switches that is a direct consequence of this control strategy. Better results can be obtained when control of the hysteresis width is used. According to [1], the width depends on the demanded switching frequency $f_{s}$ of the converter, on the inverter side filter inductance $L_{i}$, on the actual value of DC-link voltage $U_{D C}$, and on the filter capacitor voltage $u_{C f}$.

$$
H y=\left|u_{C f}\right| \frac{U_{D C}-\left|u_{C f}\right|}{L_{i} f_{s} U_{D C}}
$$

Implementation of equation (1) causes the switching frequency to remain approximately constant. The influence of variable hysteresis width is depicted in Fig. 4.

A further improvement of the output current shape involves adapting the hysteresis controller. A simple

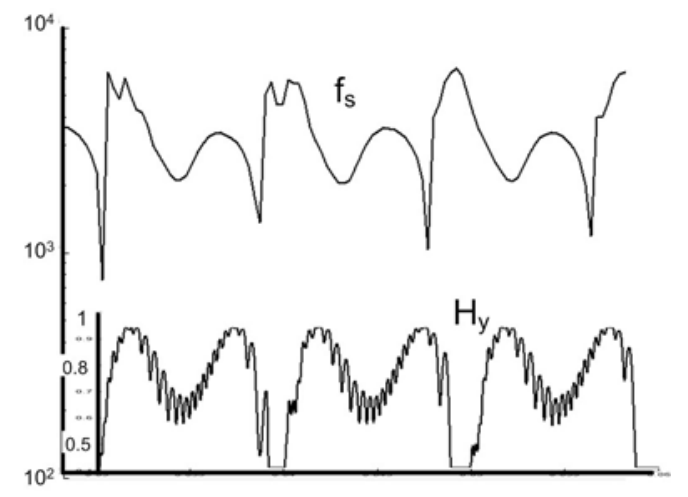

Fig. 4: Switching frequency during variable hysteresis control

hysteresis controller alternates only between two combinations $\left(S_{1}, S_{2}\right)$ or $\left(S_{3}, S_{4}\right)$, which means that either $+U_{D C}$ or $-U_{D C}$ appears on the output (Fig. 5).

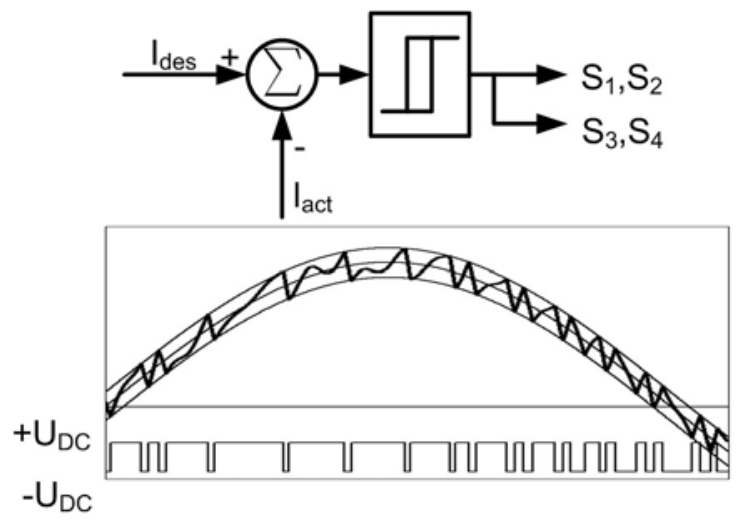

Fig. 5: Bipolar hysteresis controller

With the help of the PWM switching analogy, bipolar switching can easily be adapted to unipolar switching. This method generates three output voltage levels $+U_{D C}, 0$ and $-U_{D C}$. The controller is shown in Fig. 6. Two hysteresis controllers of different hysteresis width are used instead of one. The first controls switches $S_{2}, S_{3}$ and the second controls $S_{1}$, $S_{4}$. Outer hysteresis is used in areas where the current crosses zero and the drop in the desired current
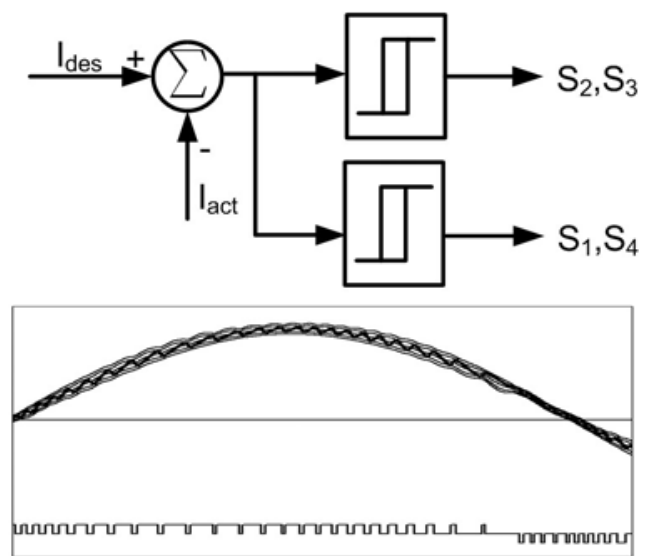

Fig. 6: Unipolar hysteresis controller 
value is faster than the drop in the output current. The output current then reaches the outer hysteresis, and the controller switches from $S_{1}$ to $S_{4}$ or vice versa.

This control of the VSI is used when the converter operates on the supply network and is used for generation of current. The output voltage is guarded by the supply network itself. The converter "pushes" current into the grid.

\subsection{PWM control}

Hysteresis control cannot operate in "island mode", because there is no supply network voltage that can guard the generated voltage. Then the converter is supposed to generate output voltage with a sinusoidal shape, as in the supply network. The PWM control algorithm was therefore used for "island mode". The controller is shown in Fig. 7. The PI controller is used in the voltage control loop. The output of the controller is the duty cycle for the modulator.

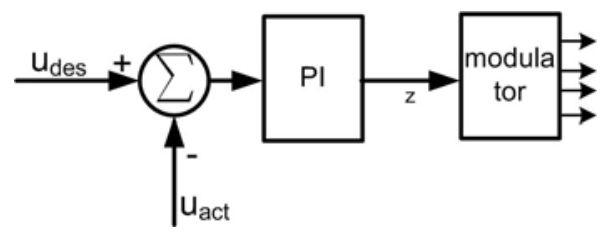

Fig. 7: Island mode control loop

\section{L-C-L filter design}

The filter is an important part of every semiconductor converter. The filter reduces the effects caused by switching semiconductor devices on other devices. The generation of the harmonics around switching frequency can be filtered by large inductance connected to the output of the converter. But the large inductance decreases the dynamics of the system and also the operation range of the converter. Instead of the inductance the inverter can be equipped with an L-C-L filter. The L-C-L filter has good current ripple attenuation even with small inductance values. However, it can bring also resonances and unstable states into the system. The filter must therefore be designed precisely according to the parameters of the specific converter. In the technical literature we can find many articles on the design of the L-C-L filters $[2,3]$.

Table 1: Parameters for calculating the filter components

\begin{tabular}{|l|r|}
\hline Grid Voltage (V) & 230 \\
\hline Output Power of the Inverter (kVA) & 1.5 \\
\hline DC link Voltage (V) & 400 \\
\hline Grid Frequency (Hz) & 50 \\
\hline Switching Frequency (Hz) & 3000 \\
\hline
\end{tabular}

Now the filter design will be described. The system parameters considered for calculating the components for a filter with power approx. $1.5 \mathrm{kVA}$ are shown in Tab. 1.

First, the base values need to be calculated. These values are later used for calculating the filter components.

$$
\begin{gathered}
Z_{b}=\frac{U_{n}^{2}}{S_{n}}=46 \Omega \\
C_{b}=\frac{1}{\omega_{n} Z_{b}}=69.91 \mu \mathrm{F}
\end{gathered}
$$

The first step in calculating the filter components is the design of the inverter side inductance $L_{i}$, which can limit the output current ripple by up to $10 \%$ of the nominal amplitude. It can be calculated according to the equation derived in [3]:

$$
L_{i}=\frac{U_{D C}}{16 f_{s} \Delta I_{L_{-} \max }}=17.7 \mathrm{mH}
$$

where $\Delta I_{L_{-} \max }$ is the $10 \%$ current ripple specified by (5)

$$
\Delta I_{L_{-} \max }=0.01 \frac{P_{n} \sqrt{2}}{U_{n}}=0.234 \mathrm{~A}
$$

The design of the filter capacity proceeds from the fact that the maximal power factor variation acceptable by the grid is $5 \%$. The filter capacity can therefore be calculated as a multiplication of system base capacitance $C_{b}$

$$
C_{f}=0.05 C_{b}=3.45 \mu \mathrm{F}
$$

The grid side inductance $L_{g}$ can be calculated as (7)

$$
L_{g}=r L_{i}=0.32 L_{i}=5.7 \mathrm{mH}
$$

The last step in the design is the control of the resonant frequency of the filter. The resonant frequency must have a distance from the grid frequency and must be minimally one half of the switching frequency, because the filter must have enough attenuation in the switching frequency of the converter. The resonant frequency for the L-C-L filter can be calculated as (8)

$$
f_{\text {res }}=\frac{1}{2 \pi} \sqrt{\frac{L_{i}+L_{g}}{L_{i} L_{g} C_{f}}}=1.30 \mathrm{kHz}
$$

In order to reduce oscillations and unstable states of the filter, the capacitor should be added with an inseries connected resistor. This solution is sometimes called "passive damping". It is simple and reliable, but it increases the heat losses in the system and it greatly decreases the efficiency of the filter. The value of the damping resistor can be calculated as (9)

$$
R_{s d}=\frac{1}{3 \omega_{r e s} C_{f}}=11.2 \Omega
$$

So-called "active damping" methods with a virtual resistor were therefore developed. As mentioned in [4], 
there are four possible ways to place a virtual resistor for active damping. One of them is shown in Fig. 8.
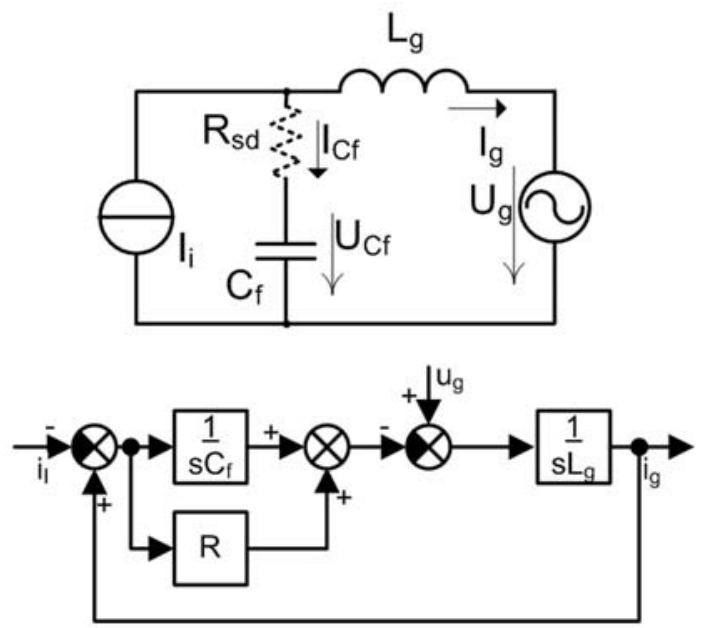

Fig. 8: A single phase L-C-L filter and an alternative circuit

Figure 8 shows a model of a single phase L-C-L filter with resistor damping connected in series with the filter capacitor. The inverter can be considered as a current source. The resistor reduces the voltage across the capacitor by a voltage proportional to the current that flows through it. In the control loop the current through $C_{f}$ is measured and differentiated by the term $s C_{f} R_{s d}$. A real resistor is not used, and the calculated value is subtracted from the demanded current (Fig. 9).

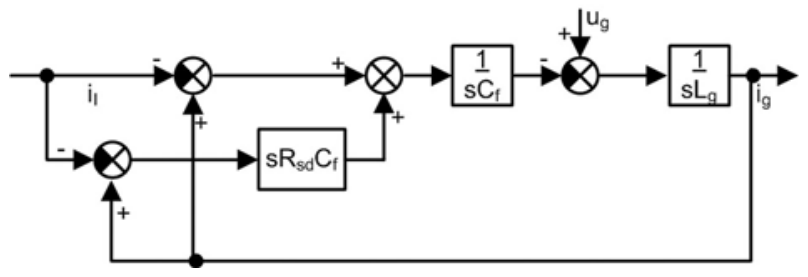

Fig. 9: A single phase L-C-L filter with a virtual resistor

In this way the filter is actively damped with a virtual resistor without losses. The disadvantage of this method is that an additional current sensor is required and the differentiator may bring noise problems because it amplifies high frequency signals.

\section{Simulation results}

A model of the VSI with the control was made with the help of Matlab-Simulink SW. All simulations were made for output current $I_{g}=3 \mathrm{~A}$, output voltage $U_{g}=230 \mathrm{~V}$ and output frequency $f=50 \mathrm{~Hz}$. The switching frequency of the inverter was $f_{s}=3 \mathrm{kHz}$ a filter with parameters designed in this paper $L_{i}=$ $17.7 \mathrm{mH}, C_{f}=3.45 \mu \mathrm{F}, L_{g}=5.7 \mathrm{mH}$ and damping resistor $R_{s d}=11.2 \Omega$ was connected to the output of the filter. Fig. 10 shows the effects of the virtual damping resistor from time $t=0.1 \mathrm{~s}$ when the filter is damped with a virtual resistor.

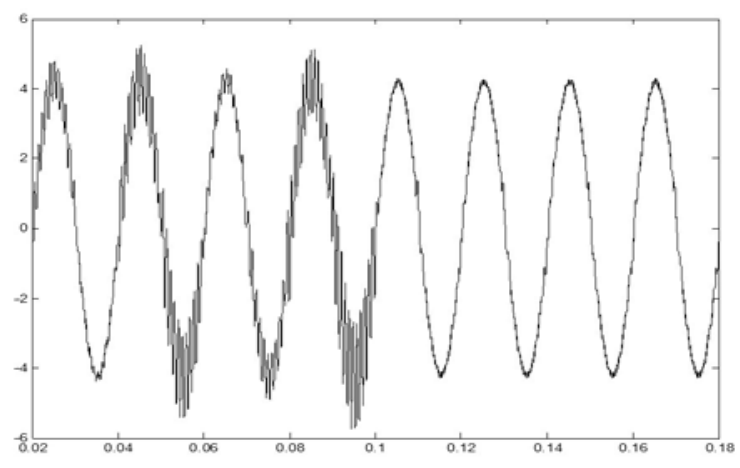

Fig. 10: Effect of the virtual resistor on the L-C-L filter

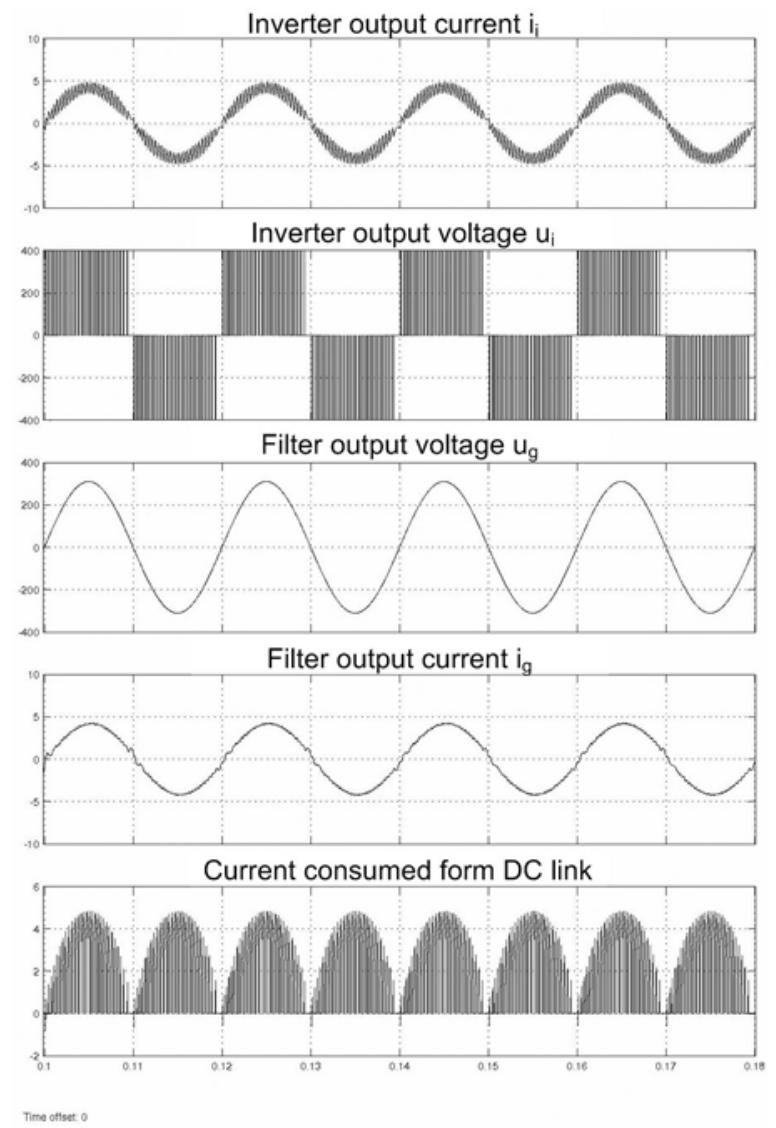

Fig. 11: Simulation results for current hysteresis control

Figure 11 shows the simulation results of the inverter with double hysteresis control. The filtered current $i_{g}$ is in the phase with the grid voltage. Except for small spikes in areas where the current changes its direction, the shape of the filtered current is sinusoidal.

The same simulations were done for "island mode" operation. In this case, the output voltage is regulated to $u_{g}=230 \mathrm{~V}$ and the current is determined by the load. The shape of the current is smoother, but there is a slight phase shift, caused by the output filter inductance and the load character. The harmonics content of both currents is good. 


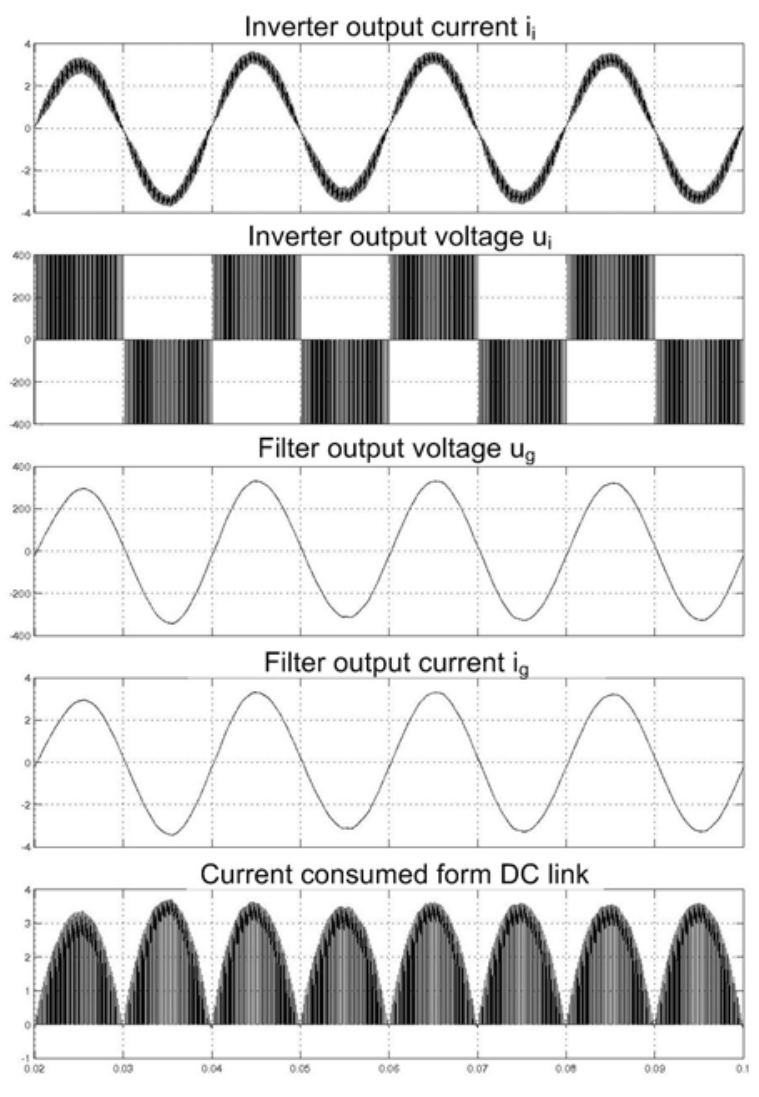

Fig. 12: Simulation results for island mode with PWM control

\section{Conclusion}

The control algorithm for a grid connected voltage source inverter has been presented here. A sinusoidal line current is produced due to the hysteresis controller. The switching frequency is almost constant because of the variable hysteresis width control. The output current filter has been designed and simulated. The obtained results seem to be promising. However, we will not be able to evaluate whole system until after it has been realized.

\section{Acknowledgement}

This work was supported by the Grant Agency of the Czech Technical University in Prague, grant No. SGS 10 800630. The research described in the paper was supervised by Prof. J. Lettl, CSc., FEE CTU in Prague.

\section{References}

[1] Hinz, H., Mutschler, P., Calais, M.: Control of a single phase three level voltage source inverter for grid connected photovoltaic systems, PCIM 1997.

[2] Liserre, M., Blaabjerg, F., Hansen, S.: Design and control of an lcl-filter based three-phase active rectifier. In Industry Applications Conference, 2001. Thirty-Sixth IAS Annual Meeting. Conference Record of the 2001 IEEE, volume 1, 2001.

[3] Araújo, S. V., Engler, A., Sahan, B.: LCL Filter design for grid-connected NPC inverters in offshore wind turbines. In The rth International Conference on Power Electronics. Daegu (Korea), 2007.

[4] Dahono, P. A.: A method to damp oscillations on the input lc filter of current-type ac-dc pwm converters by using a virtual resistor. In Telecommunications Energy Conference INTELEC'03, 2003.

\section{About the author}

Jan BAUER was born in Prague on January 14, 1983. He studied electrical engineering at CTU in Prague and was awarded his master's degree in June 2007. He is currently working on his $\mathrm{PhD}$ thesis at CTU in Prague, FEE at the department of Electric Drives and Traction. His main fields of interest are modern control algorithms for inverters and matrix converters.

Jan Bauer

E-mail: bauerj1@.fel.cvut.cz

Dept. of Electric Drives and Traction

Faculty of Electrical Engineering

Czech Technical University in Prague

Technická 2, 16627 Praha, Czech Republic 